\title{
A New Soft Material Based In-the-Ear EEG Recording Technique
}

\author{
Hao Dong ${ }^{1}$, Paul Matthews ${ }^{2}$ and Yike Guo ${ }^{3 *}$
}

\begin{abstract}
Long-term electroecephalogram (EEG) recording is important for seizure detection, sleep monitoring and etc. In-the-ear EEG device makes such recording robust to noise and privacy protected (invisible to other people). However, the state-of-art techniques suffers from various drawbacks such as customization for specific users, manufacturing difficulties and short life cycle. In this paper, we proposed a new soft material based in-the-ear EEG recording technique to address all the issues. Moreover, our techniques allows mass production with low cost.
\end{abstract}

\section{INTRODUCTION}

Scalp EEG, both wet and dry electrode [4], have been used for seizure detection, sleep monitoring and etc., [1], [2], [3]. However, long-term monitoring becomes their limitation for many practical applications since it requires privacy (invisible to the people) and wearing comfort. The in-the-ear EEG techniques makes the long-term monitoring possible. Unlike scalp EEG which attaches many electrodes and cables to a user's scalp, the in-the-ear EEG devices will bring comfortable user experience. Moreover, the EEG signal is robust to noise incurred by users' movements and other unknown sources. In this paper, we will show the advantage of our soft material based technique to address these issues.

First of all, we review three representative in-the-ear electrode techniques. The first in-the-ear EEG device was invented in 2012 [5] aiming to prove the feasibility of recording EEG in the ear. The ear-canal-fit electrode is achieved by embedding $\mathrm{Ag} / \mathrm{AgCl}$ electrodes on a customized orthoplastic earpiece. The nonconducting orthoplastic earpiece is used to hold $\mathrm{Ag} / \mathrm{AgCl}$. The evaluations include alpha attenuation response (AAR), auditory steady-state response (ASSR) [6] and visual event-related potential (VERP), which prove the concept of in-the-ear EEG is work.

However, due to the variation of shape/size of human ear canal, it would be extremely challenging to customize the ear electrodes owning to the unbend property of material. Meanwhile, the comfort issue has not been resolved.

Recent studies put advance to fabricate carbon nanotube polydimethylsiloxane (CNT/PDMS) based ear electrodes. CNT/PDMS belongs to conductive soft material that easily fits ears without customization [7]. As there is no requirement to put any holding material, soft electrode material based technique prevails to simplify the electrode structure. However, it is difficult to produce the CNT/PDMS material

Hao Dong is with Data Science Institute, Imperial College London, UK, SW7 2AZ e-mail: hd311@imperial.ac.uk

Paul Matthews is with Department of Medicine, Imperial College London, UK, SW7 2AZ e-mail: p.matthews@imperial.ac.uk

Yike Guo is with Data Science Institute, Imperial College London, UK, SW7 2AZ e-mail: y.guo@imperial.ac.uk, * corresponding author which requires several hours and high production cost for dispersion thus prevent the scalability for mass production [8], [9].

Either soft or hard materials are utilized for each method mentioned above. In [10], soft holding material is used to solve the customization problem.where memory foam is used as soft holding material and conductive cloth electrodes are used as electrode material.

The memory foam can adjust to the shape of ear canal then provide uniform pressure against the skin. On the other hand, the chosen cloth electrode is made of silver-coated nylon interwoven with elastic fibres, it is comfortable enough for long-term recording. However, the durability of both memory foam and silver-coated nylon are short, and the abrasion of cloth electrode will lead to low frequency noise. This design shows that the combination of different material to fabricate an electrode is easier than to produce soft electrode material only.

However, the limitation of in-the-ear EEG is its spatial resolution. The EEG in the ear is transmitted from the temporal lobe, where there is more information on auditory but less on other features. Thanks to the high correlation of EEG signal within one location with its surroundings [12], the information from frontal, occipital, parietal and central lobe can also be reflected and extracted from the EEG in the ear.

\section{MATERIAL}

TABLE I

FEATURES OF DIFFERENT MATERIALS

\begin{tabular}{ll}
\hline Material & Performance features \\
\hline Tin & Low precision on low frequency range \\
\hline Gold or silver plating & Abrasion can cause low frequency noise \\
\hline $\begin{array}{l}\mathrm{Ag} / \mathrm{AgCl} \text { plating on non- } \\
\text { silver material }\end{array}$ & $\begin{array}{l}\text { Abrasion can cause low frequency } \\
\text { noise, single use only }\end{array}$ \\
\hline Silver & Durable and low noise \\
\hline $\mathrm{Ag} / \mathrm{AgCl}$ plating on silver & $\begin{array}{l}\text { Fast signal stabilization, low noise, suit- } \\
\text { able for measuring evoked potential and } \\
\text { event-related potential }\end{array}$ \\
\hline Sintered $\mathrm{Ag} / \mathrm{AgCl}$ & $\begin{array}{l}\text { Fast signal stabilization, low noise, suit- } \\
\text { able for measuring evoked potential and } \\
\text { event-related potential }\end{array}$ \\
\hline
\end{tabular}

Different materials have different level of low frequency noise [11]. Typically, the material classifies the electrodes into different categories as Table I illustrated. Sintered silversilver chloride $(\mathrm{Ag} / \mathrm{AgCl})$ has the best low noise perfor- 


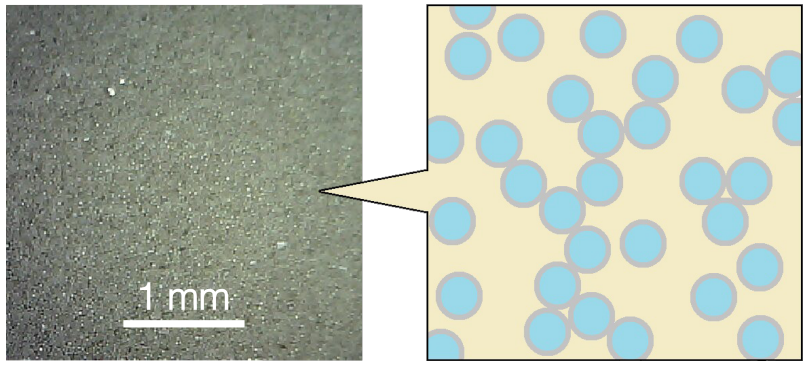

Fig. 1. Inner structure of silvered glass silicone (SGS)

mance, and $\mathrm{Ag} / \mathrm{AgCl}$ plating on silver has similar performance as well. Silver has the second best performance and lower cost compare with $\mathrm{Ag} / \mathrm{AgCl}$; so in our study, we choice silver as conductive medium for the sake of cost and mass production.

The proposed canal-type ear electrode (CEE) were fabricated in two steps. In the first step, the raw material is silvered glass beads filled silicone rubber; in other word, it is silver, glass silver and silver conductive grains distribution in silicone rubber, through the pressure of conductive particle contact, achieve good conductivity. As emphasised below, the silvered glass silicone (SGS) is silvered glass beads filled silicone rubber.

After producing the raw material, we can move forward to moulding process. The advantage of our material allow us to use conventional compression moulding to achieve a given shape. The moulding process requires $200^{\circ} \mathrm{C}, 150$ seconds and 180 tons of pressure. A multi-parts steel mould can be used repeatedly for many times, and able to product over 50 pieces in one machining cycle. Finally, the hardness of ear electrode is 60 to 70A. The finishing colour is yellow, but it can be dyed to different colour in the manufacturing process such as dark khaki, black. Figure 7 shows our inthe-ear electrode made of SGS, each SGS-CEE cost around 0.2 USD during mass production.

The advantage of SGS-CEE is easy to make. Comparing with CNT/PDMS [8], this method largely reduces the fabricate process from several hours to less than 150 seconds, which is low in cost, high speed and capable of large scale manufacturing.

\section{STRUCTURE}
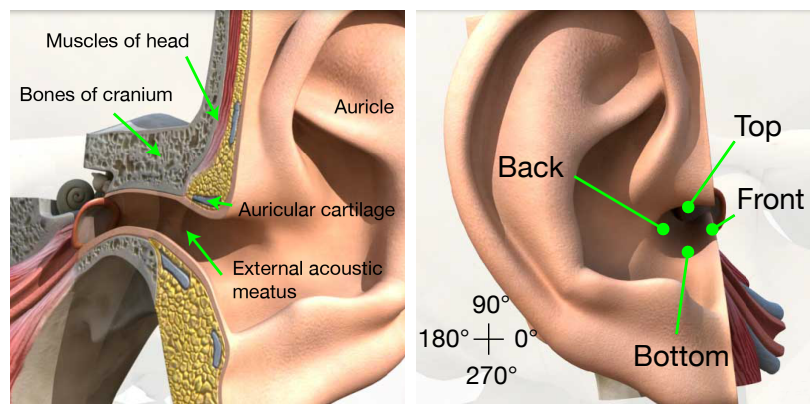

Fig. 2. Ear structure
The ear canal is surround by auricular cartilage, muscles of head, bones of cranium and fat. So the EEG from different position of ear canal are different in some degree, and uniform pressure against skin is essential.

Inside the ear canal, both the bones of cranium and auricular cartilage are able to holding the electrode, but due to the bones of cranium is hard, it will cause some pain if the electrode contact with it for a long time. In contrast, auricular cartilage is perfect for holding the electrode, it can be attributed to its flexibility and shallow depth.
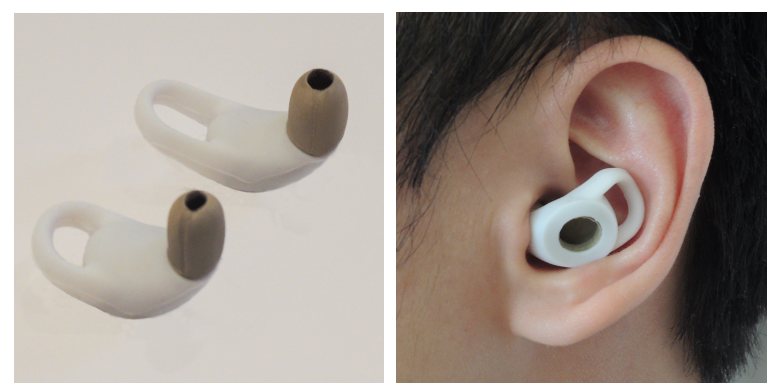

Fig. 3. SGS ear canal electrode

In order to suit people of different ear size and provide uniform pressure against skin, the SGS electrode come in different sizes. Besides, the electrode is designed to be supported by auricle as Figure 3 shows, which is common in earphone. This stabilizing structure is able to reduce the noise caused by movements. Because it is made of non-conductive silicone, no effect on EEG recording when it contact with auricle.

\section{Evaluation}

To evaluate the proposed in-the-ear electrode, we test the material and in-the-ear EEG separately. The most common test methods are observing eyes blink, gritting teeth, Alpha attenuation response (AAR, also know as alpha rhythm detection) and Auditory steady-state response (ASSR). In the evaluation, in order to get the result close to wireless and wearable application, an analog and digital circuit was designed to record the EEG in dry way, the hardware informations are described on Table II (Note: CMRR - commonmode rejection ratio, ADC - analog to digital converter, LSB - least significant bit). After convert the analog signal to digital data, It transmit the data via blue-tooth low energy technology (BLE) to an iPhone 6.

For the evaluation of material, we recorded the EEG on the scalp using a $12 \times 10 \times 1.2 \mathrm{~mm}$ SGS sheet placed between Fpz and active dry electrode (as shown in Figure 4 ), and placed the reference and DRL on the subject's left earlobe. From Figure 5, the eyes blinks and gritting teeth can be clearly visualized on the measured EEG signal, where each eyes blink will produces one peak, and each teeth gritting will produces a continuous large vibration.

In the evaluation, the subject is seating on a chair with a backrest and looking ahead under normal lighting condition is defined as neutral state. Close eyes state represents the 
TABLE II

TECHNICAL INFORMATION OF ALL-IN-ONE IN-EAR EEG SYSTEM

\begin{tabular}{ll}
\hline Parameters & Value \\
\hline CMRR & $120 \mathrm{~dB}$ \\
\hline Input impedance & $10^{12} \Omega$ \\
\hline Sampling rate & $512 \mathrm{~Hz}$ \\
\hline ADC resolution & $12 \mathrm{Bits}$ \\
\hline Frequency response & $0.2-49 \mathrm{~Hz}$ \\
\hline Voltage resolution & $0.18 \mu \mathrm{V} / \mathrm{LSB}$ \\
\hline
\end{tabular}

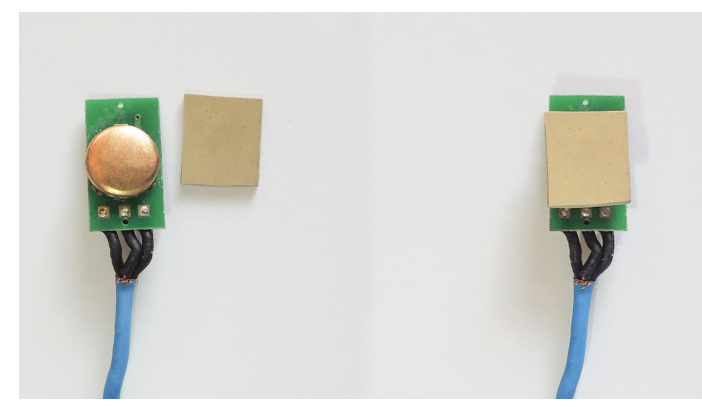

Fig. 4. Flat SGS dry electrode for evaluating the material
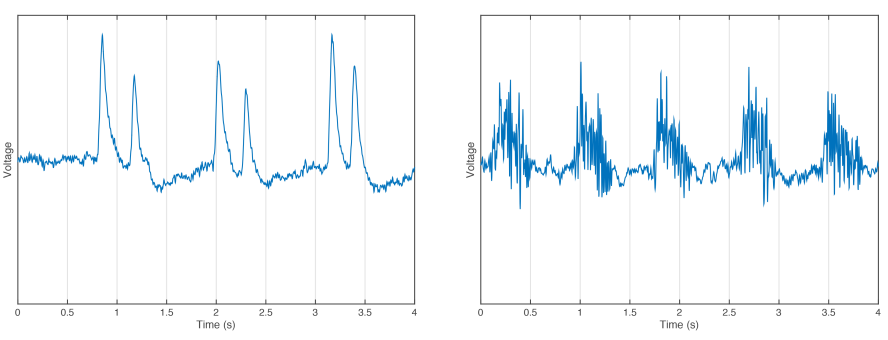

Fig. 5. Eyes blink and gritting teeth detect from Fpz using SGS sheet. Left: blinks 6 times; Right: gritting teeth 5 times
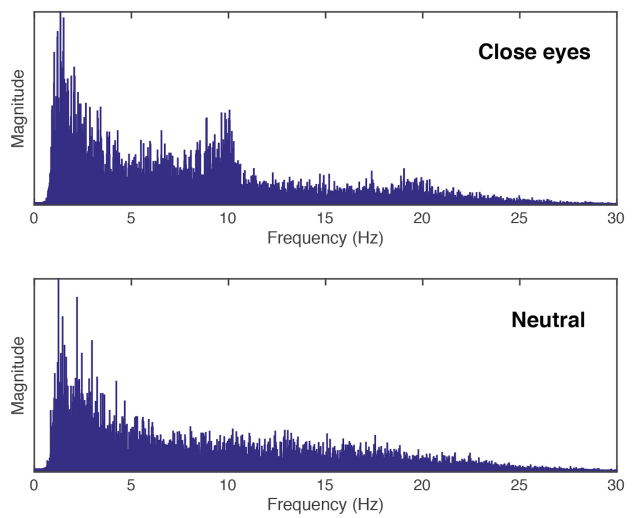

Fig. 6. Alpha attenuation response on Fpz. Upper graph shows eyes close case and lower graph shows eyes open case

subject is seating on a chair with a backrest without any movement, the same with neutral state, but eyes closing. The alpha rhythm $(8-12 \mathrm{~Hz})$ should has significant increase during close eyes period compare with neutral state.
In the AAR test, the alpha activity was successfully recorded from Fpz channel. Figure 6 shows the frequency spectrums of EEG signals during neutral state and close eyes state. It can be clearly see that the alpha rhythm $(8-12 \mathrm{~Hz})$ has significant increase during eyes closing compared to eyes opening. This test proves the SGS is suitable for dry EEG electrode.
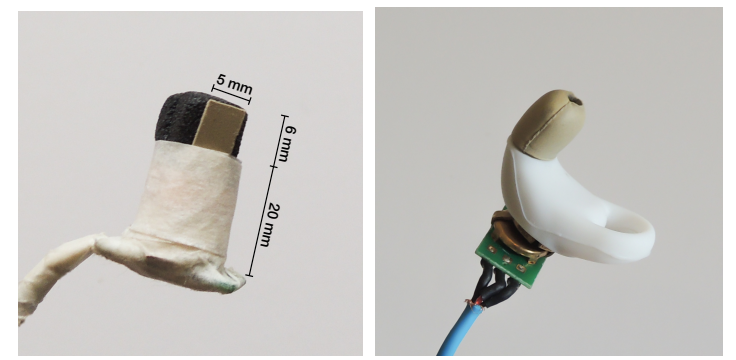

Fig. 7. SGS sheet dry electrode for testing different position (left), Canaltype SGS dry electrode with electric circuit (right)

Furthermore, to evaluating the in-the-ear EEG, the EEG signal is measured from different positions of ear canal. We measured the signals from 4 directions (shown in Figure 2): top, bottom, front and back, using the electrode shown in the left side of Figure 7. According to our experiment, the EEG from front position has artifact caused by the blood vessel on cheek, and the fluctuation of this artifact synchronizes with heartbeat. The heart information can be detected, but the EEG signal is contaminated. Moreover, the distance between bottom position and temporal lobe is larger than the other positions', thus the EEG from bottom position contains lesser EEG information than others'. Among these positions, the top position was found to has the best performance for detecting alpha activity, due to short distance to temporal lobe compare with others.

Figure 8 shows the frequency and time-frequency analysis of EEG signal measured from the top position, where the red spot on the time-frequency spectrogram represents high amplitude. It is clear that the frequency between 8 to $12 \mathrm{~Hz}$ has significant and uninterrupted increasement during eyes closing. The left hand side of Figure 8 shows the amplitude of alpha rhythm. Moreover, compare with EEG from frontal lobel, the eyes blink and gritting teeth artifacts from in-theear EEG are significantly lower.

Furthermore, to check the adaptable of using the SGS for long-term wearing, a skin compatibility test was made. To complete the test, a $15 \times 15 \times 1.2 \mathrm{~mm}$ material sheet was continuously attached on subjects' skin. The test on three participants showed no side effects of wearing it for long time, which verified the skin compatibility of the SGS. Besides, the SGS sheets are used to measure EEG after skin compatibility test, the EEG quality is unchanged after skin contact for a week. This means the skin would not cause material metamorphism.

\section{CONCLUSIONS}

Overall, the existing in-the-ear EEG electrode can be separated into three approaches: the first approach is using hard 
(A)

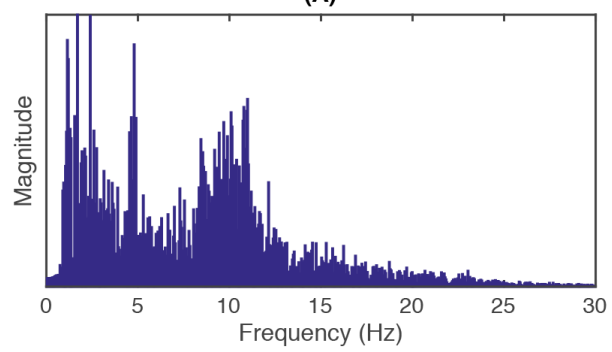

(C)

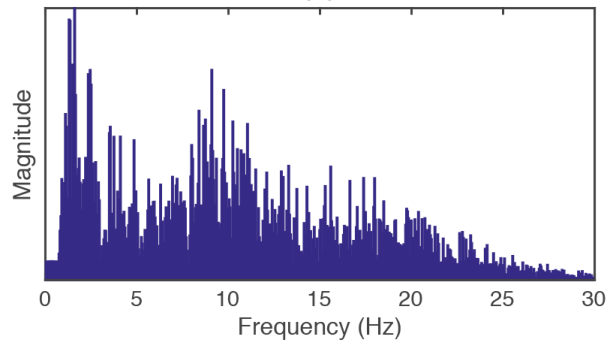

(B)

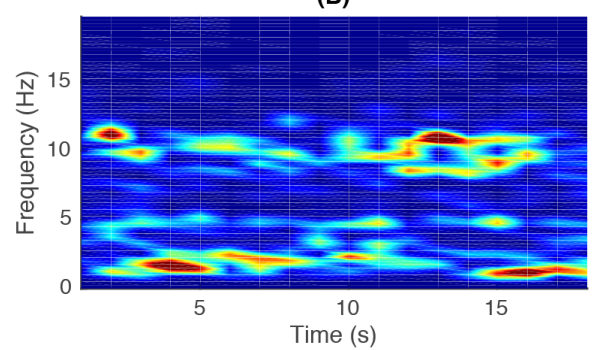

(D)

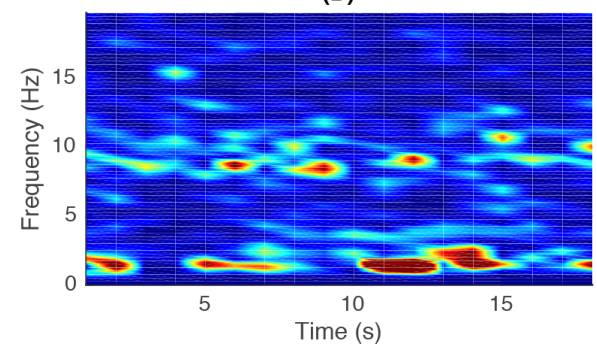

Fig. 8. Frequency spectrum (left) and time-frequency spectrogram (right) of AAR testing using SGS sheet ear canal electrode to contact with the top position of ear canal. (A) and (B) Subject A, the top position of right ear canal, reference and DRL both placed on left earlobe; (C) and (D) Subject B, the top position of right ear canal, reference and DRL both placed on left earlobe

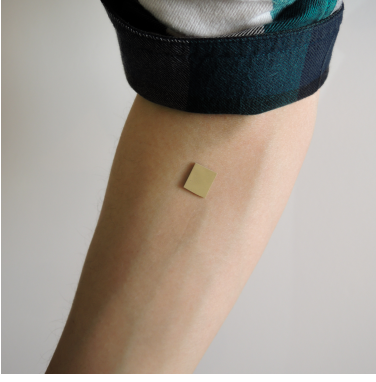

Fig. 9. Attachment of SGS sheet to the skin of upper arm for a week and show no side effects

materials only (like: $\mathrm{Ag} / \mathrm{AgCl}+$ orthoplastic); the second approach is using soft holding material (like: silver-coated nylon + memory foam); the third approach is using soft electrode material only (like: CNT/PDMS).

Obviously, the hard electrode materials have been well study. It is easy to find a hard electrode material to make an electrode according to any level of performance. In contrast, the soft electrode material has been slow to grow because the limited options of the base material (like PDMS) and the functional material (like CNT). The manufacturing issues also need to be solve before large production.

So in conclusion, to develop a practical solution in short time, combining hard or soft electrode material and soft holding material are the optimal options. For example, silicone can be adopted as holding material and solid silver as electrode material, both of these materials are durable, therefore, the electrode can be reused for many times. The challenge is the connect method between these two materials, and how to guarantee the uniform pressure against skin. The connection between two materials can be done by adhesive or physical structure joint. But in the long run, using soft electrode material without any holding material is the best approach, the silvered glass based silicone is a durable, lowcost and easy-to-make method.

\section{ACKNOWLEDGMENT}

The authors would like to thank Wei Pan, Kehan Yu and Pan Wang for helpful comments and suggestions on the manuscript.

\section{REFERENCES}

[1] Ohayon, M. M. Epidemiology of insomnia: What we know and what we still need to learn. Sleep Medicine Reviews, 6(2), 97-111. 2002.

[2] Katharina Wulff, Silvia Gatti, Joseph G Wettstein, and Russell G Foster. Sleep and circadian rhythm disruption in psychiatric and neurodegenerative disease. 11(August), 2010.

[3] Ramgopal, Sriram et al. Seizure Detection, Seizure Prediction, and Closed-Loop Warning Systems in Epilepsy. Epilepsy \& behavior: 2014.

[4] Chi, Y. M., Jung, T., \& Cauwenberghs, G. Dry-contact and Noncontact Biopotential. IEEE Reviews in Biomedical Engineering, 3, 106-119. 2010.

[5] David Looney et al. The in-the-ear recording concept: User-centered and wearable brain monitoring. IEEE Pulse, 3(6):32-42, 2012.

[6] Kidmose, P., Looney, D., \& Mandic, D. P. Auditory evoked responses from Ear-EEG recordings. Proceedings of the Annual International Conference of the IEEE Engineering in Medicine and Biology Society, EMBS, 586-589, 2012.

[7] J.H. Lee et al. CNT/PDMS-based canal-typed ear electrodes for inconspicuous EEG recording. Journal of neural engineering, 11(4):046014, 2014.

[8] Ha-chul Jung, Jin-hee Moon, Dong-hyun Baek, Jae-hee Lee, Yoonyoung Choi, and Joung-sook Hong. CNT / PDMS Composite Flexible Dry Electrodes for Long-Term ECG Monitoring. 59(5):1472-1479, 2012.

[9] Joung Sook Hong and Chongyoup Kim. Extension-induced dispersion of multi-walled carbon nanotube in non-Newtonian fluid. Journal of Rheology, 51(5):833, 2007.

[10] Goverdovsky, V., Looney, D., Kidmose, P., \& Mandic, D. In-ear EEG from viscoelastic generic earpieces: Robust and unobtrusive 24/7 monitoring. IEEE Sensors Journal, 2015. 
[11] P. Tallgren, S. Vanhatalo, K. Kaila, and J. Voipio. Evaluation of commercially available electrodes and gels for recording of slow EEG potentials. Clinical Neurophysiology, 116:799-806, 2005.

[12] Mike X Cohen. Analyzing Neural Time Series Data: Theory and Practice. The MIT Press. 